\title{
Turning-rate Selective Control : A New Method for Independent Control of Stress-engineered MEMS Microrobots
}

\author{
Igor Paprotny ${ }^{* \dagger * * \dagger \dagger}$, Christopher G. Levey ${ }^{\ddagger}$, Paul K. Wright ${ }^{\dagger \S}$ and Bruce R. Donald $\|^{\| * *}$ \\ ${ }^{*}$ Berkeley Sensor \& Actuator Center (BSAC), University of California, Berkeley, CA, USA. \\ ${ }^{\dagger}$ Center for Information Technology Research in the Interest of Society (CITRIS), University of California, Berkeley, CA, USA. \\ ¥Thayer School of Engineering, Dartmouth College, Hanover, NH, USA. \\ $\S$ Department of Mechanical Engineering, University of California, Berkeley, CA, USA. \\ IDepartment of Computer Science, Duke University, Durham, NC, USA. \\ "Department of Biochemistry, School of Medicine, Duke University Medical Center, Durham, NC, USA. \\ ${ }^{* *}$ Duke Institute for Brain Sciences, Duke University Medical Center, Durham, NC, USA. \\ $\dagger^{\dagger}$ Email: igorpapa@eecs.berkeley.edu
}

\begin{abstract}
We present a novel method for independently controlling multiple stress-engineered MEMS microrobots (MicroStressBots) through a single, global, control signal. Called Turning-rate Selective Control (TSC), this new technique employs designed variations in turning rates between individual microrobots to differentiate their motion. Despite all robots moving simultaneously and being identical except for exhibiting different turning rates, TSC can individually and independently position the robots' centers of rotation within a planar configuration space. This allows the individual robots to be independently maneuverable to within a distance equal to the turning radius (approximately half of a microrobot width) away from an arbitrary location (configuration excluding rotation) in $\mathbb{R}^{2}$. We introduce the theory behind TSC and, by using fabricated microrobots, show experimental results that confirm the feasibility of TSC for controlling multiple MicroStressBots through a single, global, control signal. We conclude by discussing how TSC can extend the maximum number of independently controllable MicroStressBots beyond previously published approaches.
\end{abstract}

\section{INTRODUCTION}

The last decade has seen the development of several novel microscale mobile robotic systems, such as electrostaticallydriven stress-engineered MEMS microrobots (MicroStressBots) [5], resonating stepping robots [9], stick-slip magnetic walkers [8], and microscrew-based swimmers [10]. Virtually all envisioned microrobotic applications (for example neurological; [1]) rely on the combined actions of many microrobots. However, control of many microrobots through a single global control signal has been demonstrated by only a few groups [6, 4]. Simultaneous control of several microrobots is significantly more challenging than control of single microrobots because one must overcome high level of underactuation present in such systems. The high level of underactuation is a result of the limited ability of the microrobots to decode a global control signal.

Donald et al. [7] analyzed the control voltage complexity of stress-engineered MEMS microrobots, defined as the number of distinct voltage levels of the control waveform required to independently maneuver $n$ robots. It was shown that MicroStressBots can exhibit sub-linear control voltage complexity $(\mathrm{O}(\sqrt{n}))$ if their steering arms are designed to have Symmetric Electromechanically Saturated (SESat) hysteresis gaps. In this work, we report on an new method to independently maneuver (independently control) MicroStressBots on a planar substrate. Called Turning-rate Selective Control (TSC), this method allows for independent control of microrobots that are only differentiated by their turning rates. Because MicroStressBots can be designed to have different turning rates, TSC fits within the paradigm of Global Control Selective Response (GCSR), where independent control of multiple microrobots is enabled by engineering the robots to exhibit different behavior during portions of the global control signal. Our TSC idea is very simple, yet highly effective, as our experimental results show (Sec. IV). The mechanism of TSC is both different and independent of the mechanism of SESat-based control, allowing TSC to be used to complement SESat-based control and increase the maximum number of independently controllable MicroStressBots.

We present the concept behind TSC, and show experimental results confirming the feasibility of TSC for enabling independent control of stress-engineered MEMS microrobots. There are no simulations in this paper; as described below, the presented control method was validated (up to proof-ofconcept) using physically fabricated MicroStressBots.

The paper is structured as follows: in Sec. II. we introduce the design, the kinematics, and notation for MicroStressBots. Sec. III describes the theory behind TSC, while experimental results confirming its feasibility are presented in Sec. IV. A concluding discussion summarizing the benefits and limitations of TSC is included in Sec. V. 


\section{Stress-engineEREd MEMS Microrobot (MicroSTRESSBOT)}

Scanning-electron micrograph (SEM) of a MicroStressBot is shown in Fig. 11 The robot is approximately $260 \mu \mathrm{m} \times 60$ $\mu \mathrm{m} \times 10 \mu \mathrm{m}$ in size, and has two actuated internal degrees of freedom; an untethered scratch-drive actuator (USDA) that provides forward motion, and a steering-arm actuator that determines whether the robot moves in a straight-line or turns. The steering arm actuator consists of a cantilever beam (80$150 \mu \mathrm{m}$ long) with a circular pad at its end (20-50 $\mu \mathrm{m}$ in diameter). The robot chassis, including the USDA and the steering arm actuator, is fabricated from polycrystaline silicon using a MEMS foundry process [3]. Post-processing is used to add a stress-layer to curve the initially planar steering arm out-of-plane. The stressor layer (consisting of evaporated chromium) is lithographically patterned to produce the exact amount of steering-arm deflection.

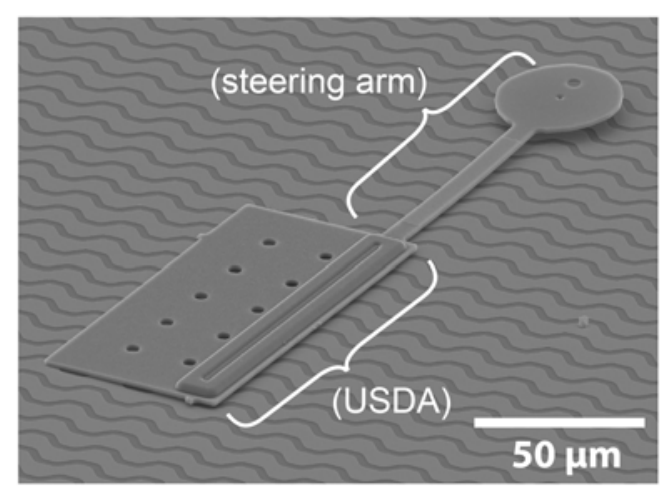

Fig. 1. SEM micrograph of a MicroStressBot. The robot consists of an untethered scratchdrive actuator (USDA) that provides forward motion (propulsion), and a steering-arm actuator that controls whether the robot moves in straight-line or turns.

MicroStressBots are actuated on a field of zirconia-insulated interdigitated electrodes. When a voltage waveform is applied between the opposing pairs of these electrodes, alternating electric potential is capacitively coupled to the microrobot chassis supplying both power for the actuation of the USDA (propulsion) and a control signal for the actuation of the steering arm. An alternating voltage waveform containing both a steering arm control signal and an USDA power-delivery waveform is called a control primitive [6]. When the steering arm is elevated and the USDA receives power, the robot moves along a straight-line trajectory. When the steering arm is lowered as the USDA is powered, the robot turns with a turning rate $\omega$ around its center of rotation, $c_{r}$.

The kinematics of the MicroStressBots are summarized in Fig. 2. Although a single robot is globally controllable within its $\mathbb{R}^{2} \times \mathbb{S}^{1}$ configuration space (C-space) [11], the robot is not small-time locally controllable, since it can only turn to one side and cannot back up. MicroStressBots can be either right- or left-handed, depending on whether the steering-arm is attached to the right of left side of the USDA, respectively.

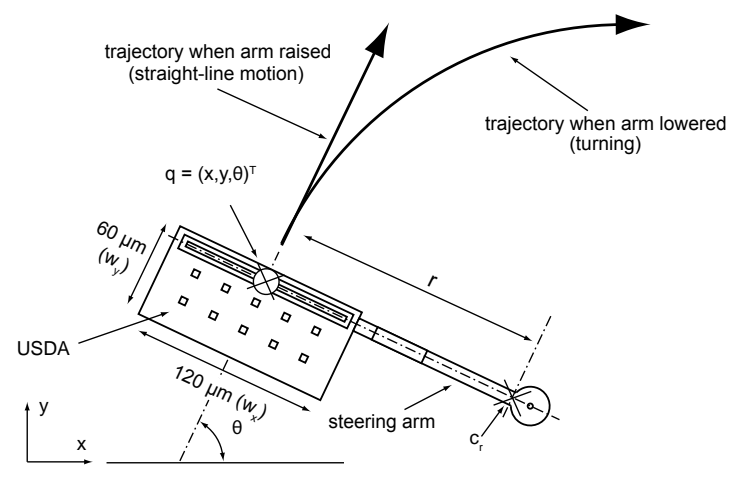

Fig. 2. Kinematics of the stress-engineered MEMS microrobot. When the steering arm is lowered, the robot turns around a center of rotation $c_{r}$.

\section{A. Turning Mechanism}

The interaction between the steering arm and the underlying substrate as the USDA is powered causes the MicroStressBots to follow a curved trajectory, i.e. to turn. The turning mechanism is illustrated in Fig. 3 During pull-down, a portion $s$ of the steering arm comes into flat contact with the substrate (Fig. 3.a). When the USDA is subsequently actuated, $s$ acts as a temporary anchor, restricting the motion of the tip of the steering arm. The robot follows a curved trajectory, flexing the steering arm until the restoring force of the arm equals the force applied by the USDA (Fig. $3 \mathrm{~b}$ ). When the arm is released during periodic polarity reversal of the $\mathrm{AC}$ waveform, the flexure in the arm is relieved, resulting in a net change in the heading $\theta$ of the microrobot (Fig. 3 b). The amount of the steering arm flexure is highly dependent on the geometry of the steering arm actuator, making the corresponding turning rate design-specific.

\section{B. Notation for MicroStressBot Control}

Consider a system of $n$ MicroStressBots $R_{i}, i \in\{1, \ldots, n\}$. Let $P_{j}$ be a control primitive, drawn from an alphabet $\Sigma$ of $m$ available control primitives; $\Sigma=\left\{P_{1}, \ldots, P_{m}\right\}$. Application of each control primitive $P_{j}$ to the power delivery substrate will cause all the MicroStressBots on that substrate to move simultaneously. However, the differences in the designs of their steering-arm actuators may cause individual robots to move along different trajectories (exhibit different behavior).

Because each robot $R_{i}$ can exhibit different behavior during each control primitive $P_{j}$, we use a control matrix to define the relationship between the applied control primitives and the resulting microrobot behavior. An $m \times n$ control matrix $M=\left(a_{j i}\right)$, where $a_{j i}$ is a pair containing the velocity $v_{j i}$ and the turning radius $r_{j i}$ of robot $R_{i}$ during the application of control primitive $P_{j}, a_{j i}=\left(v_{j i}, r_{j i}\right)$. If robot $R_{i}$ moves forward in straight line during the application of $P_{j}, r_{j i}$ approaches infinity. While turning, the velocity $v_{j i}$ and turning radius $r_{j i}$ does not vary much between the control primitives 


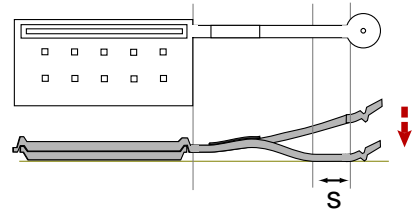

(a)

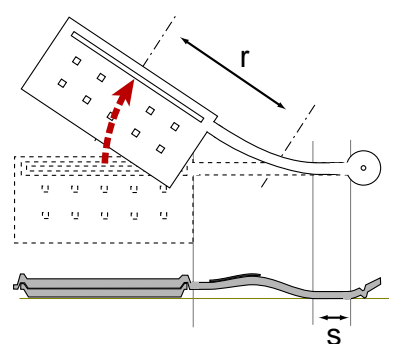

(b)

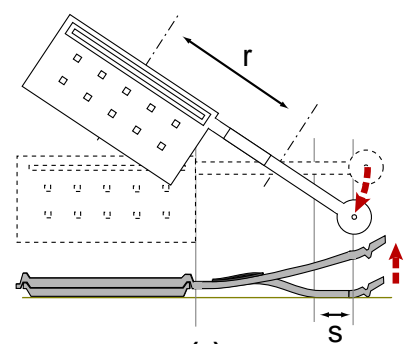

(c)

Fig. 3. Turning mechanism of the MicroStressBot (top and cross-section views). (a) The steering arm is pulled in contact with the substrate, and a flat region $s$ is temporarily anchored in place by electrostatic forces. (b) The USDA is actuated, causing the steering arm to flex while the robot follows a trajectory with the radius of curvature $r$. (c) As the arm is released during a polarity reversal of the drive voltage waveform, the flexure of the steering arm is released. This cycle is continuously repeated, causing the robot to turn.

that cause turning, and for the remainder of this paper we shall assume that $v_{i}=v_{j i}$ and $r_{i}=r_{j i}$ for all $j$ that correspond to turning control primitives. The turning rate of the robots is consequently $\omega_{i}=\frac{v_{i}}{r_{i}}$. We define the center of rotation $c_{r}$ of robot $R_{i}$ as $c_{r}\left(R_{i}\right)$.

To maneuver the microrobots on the power delivery substrate, control primitives will be applied in some sequence to actuate the robots. All the MicroStressBots on the substrate receive the same single global power-delivery and control signal, and all the robots will move during the application of the control primitives. We define a control sequence $S$ to be a sequence of control primitives

$$
S=\left\{\mathcal{P}_{1}^{t_{1}}, \mathcal{P}_{2}^{t_{2}}, \ldots, \mathcal{P}_{k}^{t_{k}}\right\},
$$

where $\mathcal{P}_{l}^{t_{l}}, l \in\{1, \ldots, k\}$, is a control primitive drawn from the alphabet of available control primitives $\Sigma$, and $t_{l}$ denotes the duration the voltage waveform described by the control primitive is applied to the substrate. Consequently, $S$ defines nominal (i.e. error free) trajectories $T_{i}, i \in\{1, \ldots, n\}$ for all $n$ microrobots on the substrate. The nominal trajectories described by the control sequence $S$ are especially useful for planning the motion of microrobots. The relationship between the control sequence and the trajectories of a system of three MicroStressBots subject to a hypothetical control sequence $S$ and a control matrix $M$ is illustrated on Fig. 4

An orbit is a trajectory that returns the microrobot to its initial configuration. An example of a circular orbit is illustrated on Fig. 4. A $c_{r}$-orbit is a trajectory that returns the center of rotation of a microrobot to its initial location. All orbits are $c_{r}$-orbits, but the inverse is not necessary the case.

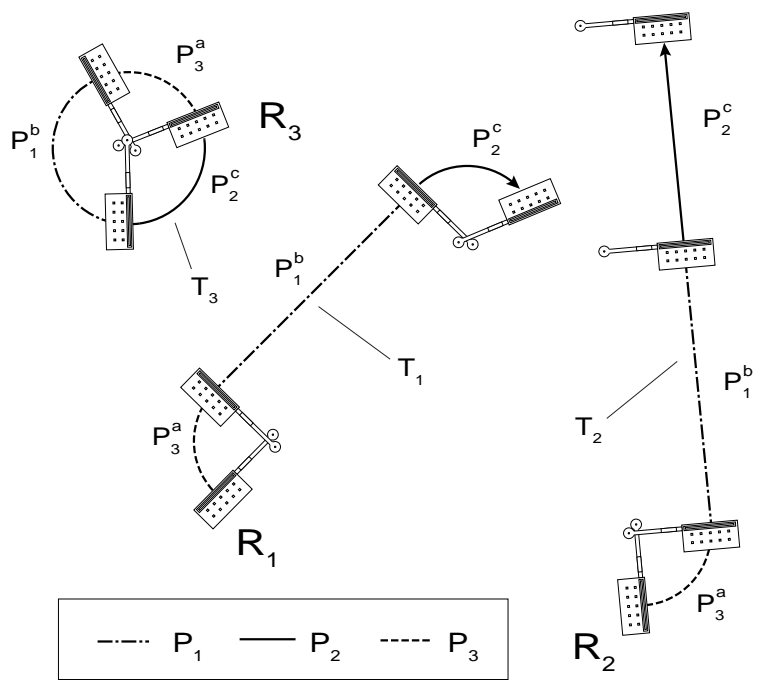

Fig. 4. Relationship between the control sequence $S$ and trajectories for a group of three microrobots, $R_{1}, R_{2}$, and $R_{3}$. Nominal trajectories $T_{1}, T_{2}$, and $T_{3}$ for a control sequence $S=\left\{P_{3}^{a}, P_{1}^{b}, P_{2}^{c}\right\}$ are shown. Note that the behavior of $R_{1}$ and $R_{2}$ only differ during the application of control primitive $P_{2}$, while robot $R_{3}$ always turns, completing an orbit.

\section{Interpolated Turning}

Interpolated turning is a technique that allows a MicroStressBot to follow a curved trajectory with an effective radius of curvature $\left(r^{\prime}\right)$ that can be set to any value between the turning radius $(r)$ and infinity by interleaving straightline and turning trajectory segments. The concept of interpolated turning is illustrated in Fig. 5. Let an interpolated turning trajectory $T_{I}$ be defined by the control sequence $S_{I}=\left\{P_{1}^{a}, P_{2}^{b}, \cdots, P_{1}^{a}, P_{2}^{b}\right\}$, where $P_{1}$ and $P_{2}$ are straightmotion and turning control primitives, respectively. Let $\rho_{a}$ and $\rho_{b}$ be the fraction of the time primitives $P_{1}$ and $P_{2}$ are applied in the control sequence $S_{I} ; \rho_{a}=\frac{a}{a+b}$ and $\rho_{b}=\frac{b}{a+b}$.

The the trajectory $T_{I}$ will now approximate a turning trajectory with a radius of curvature $r^{\prime}, r \leq r^{\prime}<\infty$, according to

$$
r^{\prime}=r\left(1+\frac{\rho_{a}}{\rho_{b}}\right) .
$$

The approximation improves as the number of primitive pairs $P_{1}-P_{2}$ repeated within $S_{I}$ increases. In our experiments, 


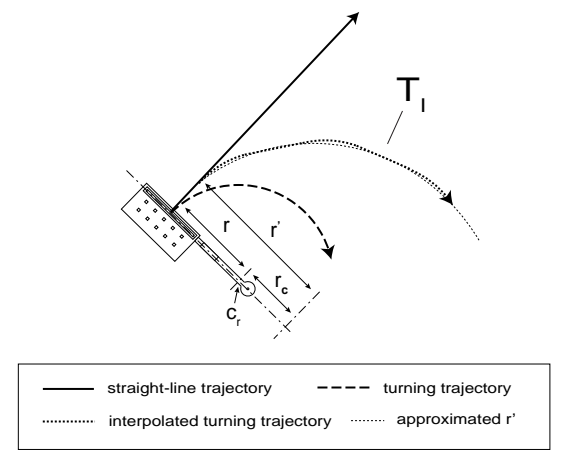

Fig. 5. Interpolated turning. The robot follows an interpolated trajectory $T_{I}$ defined by the control sequence $S_{I}=\left\{P_{1}^{a}, P_{2}^{b}, \cdots, P_{1}^{a}, P_{2}^{b}\right\}$, where $P_{1}$ and $P_{2}$ are straight-motion trajectory and turning control primitives, respectively. $\rho_{a}=\frac{a}{a+b}$ and $\rho_{b}=\frac{b}{a+b}$, and the trajectory of $S_{I}$ approximates a turning trajectory with turning radius $r^{\prime}$ according to Eq. 2).

the $P_{1}-P_{2}$ pair was merged into a new control primitive where the pair was repeated more than 20 times producing a good approximation to $r^{\prime}$.

\section{Turning-RATE Selective Control (TSC)}

The ability to vary the turning rates by varying the steering arm designs, as well as to adjust the effective turning radius $r^{\prime}$ during interpolated turning allows us to propose a new method for independent control of MicroStressBots. Called Turning-rate Selective Control (TSC), this method uses a novel mechanism to achieve independent control of MicroStressBots different from our previous presented approaches. In TSC, the microrobots are differentiated only through design-induced variation of their turning rates and not through selective pulldown and release of their steering-arm actuators [6]. This implies that that during TSC, all the robots are either turning or moving straight at any given time.

TSC is based on engineering orbit trajectories that maneuver some robots towards goal, while other robots are returned to their starting configurations. By combining several orbits, TSC can independently translate the centers of rotation $c_{r}$ of the individual robots in $\mathbb{R}^{2}$.

Consider two orbits that follow a turning trajectory and an interpolated turning trajectory, shown in Figs. $6 \mathrm{a}$ and $6 \mathrm{~b}$, respectively. When the robot is turning, the center of rotation $c_{r}$ remains in the same location in $\mathbb{R}^{2}$, as illustrated in Fig. 6a. However, when the robot is following an interpolated turning trajectory, the center of rotation $c_{r}$ is translated along a curved path with radius of curvature $r_{c}=r^{\prime}-r$. When the robot completes this orbit, $c_{r}$ returns to its starting location (Fig. 6.b).

Because each MicroStressBot makes a full rotation when it completes an orbit, MicroStressBots with different turning rates will complete their orbits at different times. Robots that are maneuvered along interpolated turning trajectories and do not complete an orbit are translated in C-space. Fig. 7 illustrates the concept of TSC. Consider robots $R_{1}$ and $R_{2}$ designed such that $\omega_{2}=\frac{1}{3} \omega_{1}$. An orbit for $R_{1}$ based on an

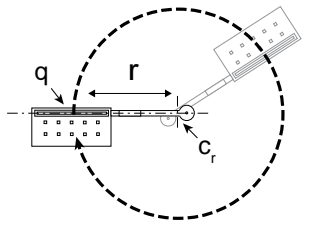

(a) (b)

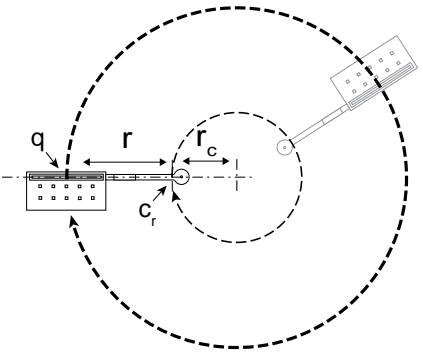

Fig. 6. Orbit trajectories. (a) During an orbit following a turning trajectory, the microrobot rotates with radius $r$ around its center of rotation $c_{r}$. The location of $c_{r}$ does not change (the orientation $\theta$ does change; $c_{r}$ is turning in place). (b) During an orbit based on an interpolated turning trajectory, the microrobot follows a trajectory with a radius of curvature $r^{\prime}=r+r_{c}$. The center of turning $c_{r}$ follows a circular trajectory (its own orbit) with radius $r_{c}$, and returns to its starting location.

interpolated turning trajectory translates the center of rotation $\left(c_{r}\left(R_{2}\right)\right)$ for robot $R_{2}$ by a vector $\gamma$. The vector $\gamma$ can be expressed as

$$
\gamma=r_{c}\left(\begin{array}{c}
\sin \theta_{2}-\sin \left(\theta_{2}+2 \pi h \frac{\omega_{2}}{\omega_{1}}\right) \\
\cos \left(\theta_{2}+2 \pi h \frac{\omega_{2}}{\omega_{1}}\right)-\cos \theta_{2}
\end{array}\right),
$$

where $\theta_{2}$ is the orientation of robot $R_{2}, h$ denotes the side the steering-arm is attached to ( $h=1$ when the robot is lefthanded, $h=-1$ when robot is righthanded), $r_{c}=r_{2}^{\prime}-r_{2}$, where $r_{2}$ and $r_{2}^{\prime}$ are the turning radius and the interpolated turning radius (from Eq. 2), respectively, of robot $R_{2}$, and $\omega_{1}$ and $\omega_{2}$ are the turning rates of robots $R_{1}$ and $R_{2}$, respectively. Note that in Fig. 7 as well as the other figures in this section, the length of the trajectories is not to scale. The starting and final locations of the centers of rotation $c_{r}$ for the robots are marked by green and yellow dots, respectively. Overlapping dots indicate no change in location. In addition, where appropriate, grey color denotes past trajectories and intermediate configurations.
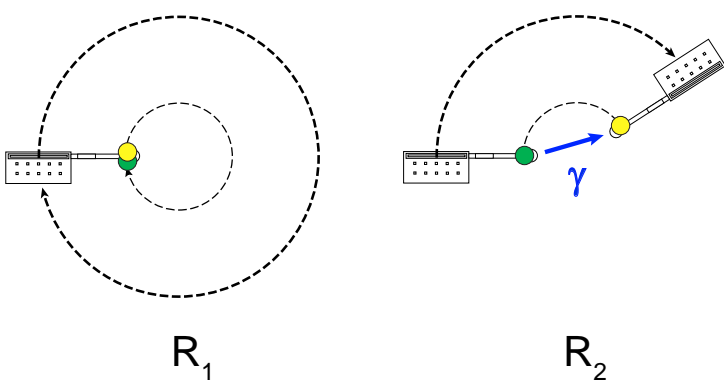

Fig. 7. The concept of Turning-rate Selective Control (TSC). Trajectories of two robots $R_{1}$ and $R_{2}$ with different turning rates: $\omega_{2}=\frac{1}{3} \omega_{1}$. As $R_{1}$ follows a circular orbit trajectory based on interpolated turning, the center of rotation $\left(c_{r}\left(R_{2}\right)\right)$ for robot $R_{2}$ is displaced by vector $\gamma$. 
After translating the center of rotation $c_{r}\left(R_{2}\right)$ by displacement vector $\gamma, c_{r}\left(R_{2}\right)$ can be translated by a second linearly independent displacement vector $\gamma_{2}$ (see Fig. 8). The vectors $\gamma$ and $\gamma_{2}$ effectively allow $c_{r}\left(R_{2}\right)$ to be translated anywhere within $\mathbb{R}^{2}$ without moving the center of rotation $c_{r}\left(R_{1}\right)$.
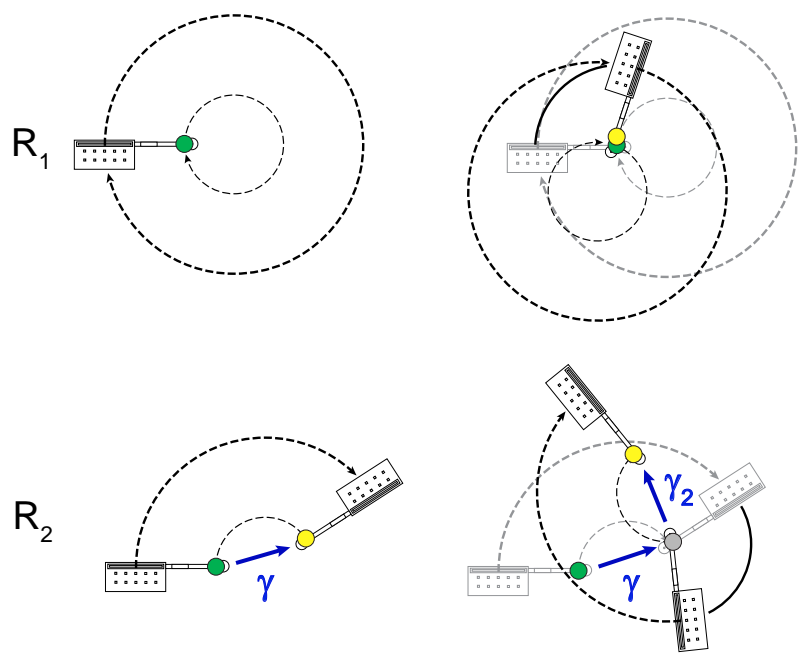

(a)

(b)

Fig. 8. Translating the center of rotation $c_{r}\left(R_{2}\right)$ by two displacement vectors that span $\mathbb{R}^{2}$. (a) Translating $c_{r}\left(R_{2}\right)$ by vector $\gamma$, while robot $R_{1}$ completes an orbit. (b) Both robots turn (non-interpolated) to an arbitrary angle, and $c_{r}\left(R_{2}\right)$ is translated by another vector $\gamma_{2}$, while robot $R_{1}$ completes another orbit. The angle between the vectors, as well as their length, can be varied arbitrary, allowing $c_{r}\left(R_{2}\right)$ to be translated in $\mathbb{R}^{2}$.

Correspondingly, the center of rotation $c_{r}\left(R_{1}\right)$ of robot $R_{1}$ can be translated while robot $R_{2}$ completes an orbit, and $c_{r}\left(R_{2}\right)$ returns to its starting location. One approach is to initially move robot $R_{2}$ using interpolated turning, and then construct a non-orbit trajectory that maneuvers $c_{r}\left(R_{2}\right)$ back to its original location, while displacing robot $R_{1}$ by vector $\eta$. This approach is illustrated in Fig. 9

\section{A. Extension to $n$ Microrobots}

TSC can be extended to independently control $n$ MicroStressBots by individually translating a single robot while the remaining $n-1$ robots complete their orbits. Consider adding a third microrobot $R_{3}$ with turning rate $\omega_{3}=\frac{2}{3} \omega_{1}$ to the system shown on Fig. 7. The center of rotation $c_{r}\left(R_{3}\right)$ and not $c_{r}\left(R_{2}\right)$ is translated by engineering the displacement vectors $\gamma$ and $\gamma_{\mathbf{2}}$ such that they cancel each other, resulting in a non-circular $c_{r}$-orbit returning the center of rotation $c_{r}\left(R_{2}\right)$ to its starting location. Because all the trajectories complete orbits for robot $R_{1}$, its center of rotation, $c_{r}\left(R_{1}\right)$, also remains in its starting location. The center of rotation $c_{r}\left(R_{3}\right)$ is translated by a vector $\zeta_{\mathbf{3}}$. The trajectories for this implementation of TSC on three robots are shown on Fig. 10 By induction, two displacement vectors $\zeta_{3}$ and $\zeta_{3}^{\prime}$ can be combined to form a $c_{r}$-orbit for robot $R_{3}$, allowing the translation of the center
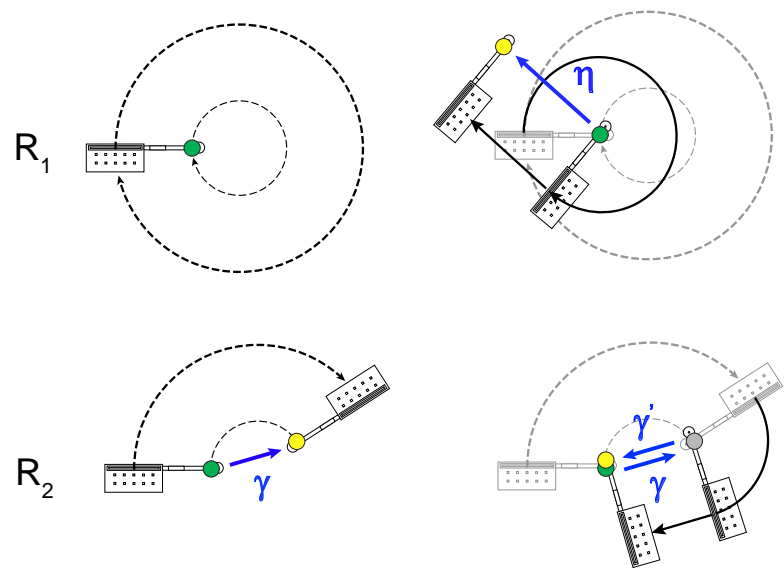

(a)

(b)

Fig. 9. Translating robot $R_{1}$ and not robot $R_{2}$. (a) Initially, the center of rotation $c_{r}\left(R_{2}\right)$ is translated by vector $\gamma$ using an interpolated turning trajectory while robot $R_{1}$ completes an orbit. (b) Both robots are then rotated such that center of rotation $c_{r}\left(R_{2}\right)$ can return to its starting location using straight-line motion along vector $\gamma^{\prime}$ (bottom), while robot $R_{1}$ and center of rotation $c_{r}\left(R_{1}\right)$ are both translated by vector $\eta$ (top).

TABLE I

EXPERIMENTALLY MEASURED TURNING RATES $\omega$ AND TURNING RADII $r$ FOR FABRICATED MICROROBOTS $R_{1}$ AND $R_{2}$

\begin{tabular}{|c||c|c|}
\hline & $\omega[\mathrm{rad} / \mathrm{s}]$ & $r[\mu \mathrm{m}]$ \\
\hline $\mathbf{R}_{1}$ & $0.46(0.05)$ & $136.66(7.6)$ \\
\hline $\mathbf{R}_{\mathbf{2}}$ & $0.20(0.01)$ & $177.50(5.0)$ \\
\hline
\end{tabular}

of rotation of a fourth robot $R_{4}$, and so on. We will use the shorthand $\operatorname{TSC}(n)$ to denote TSC of $n>2$ microrobots.

\section{EXPERIMENTAL RESULTS}

We fabricated two MicroStressBots designed to exhibit different turning rates. The designs and optical micrographs of the two robots are shown in Figs. 11 and 12 respectively. $L$ and $w$ correspond to the length and width of the steering arm, respectively. $d$ is the diameter of the steering-arm pad and $c$ is the length of the stressor layer applied to the steering arm.

The large difference in the turning rate of the robots was experimentally verified. Measured average turning rates $\omega$ and turning radii $r$ for both robots are shown in Tab. I Values in parentheses show the standard deviations. Differences of their turning rates are also clearly visible in the optical micrographs in Fig. 13. The four micrographs were taken at times $t=$ $2,6,8,12$ seconds from the start of the experiment and include traces of the completed trajectories (black) for the two robots turning.

Fig. 14 shows the basic implementation of TSC on MicroStressBots $R_{1}$ and $R_{2}$, where one of the robots is displaced while the second robot orbits back to its initial configuration, analogous to the concept illustrated in Fig. 7 The center of rotation $c_{r}\left(R_{2}\right)$ is translated by vector $\gamma$ using a interpolated 

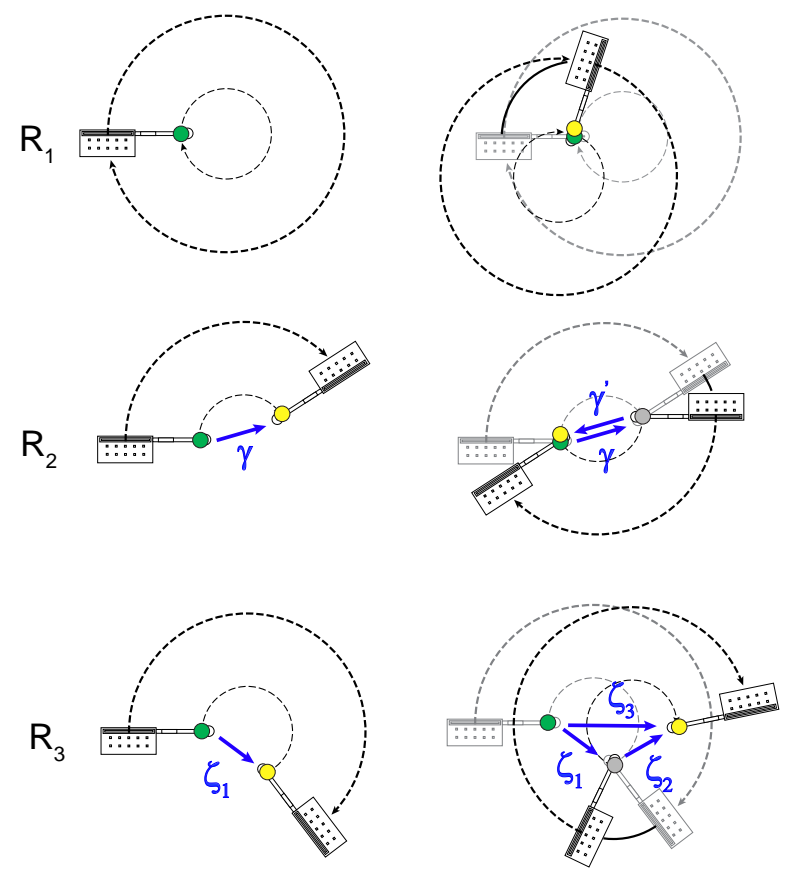

(b)

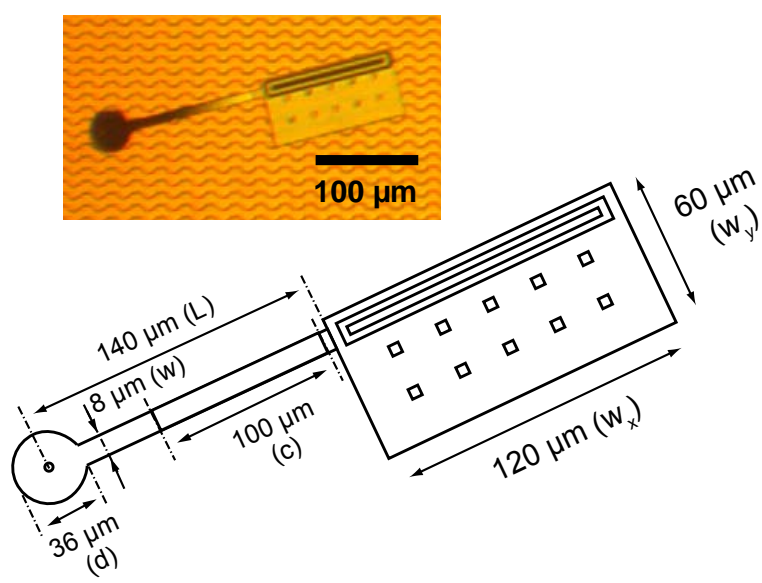

Fig. 12. Design and optical micrograph (inset) of robot $R_{2}$.

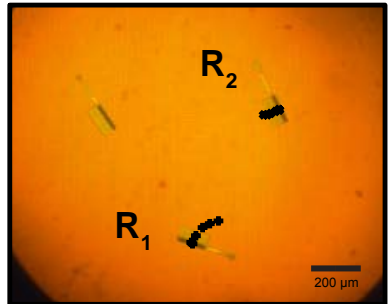

$\mathrm{t}=2 \mathrm{~s}$.

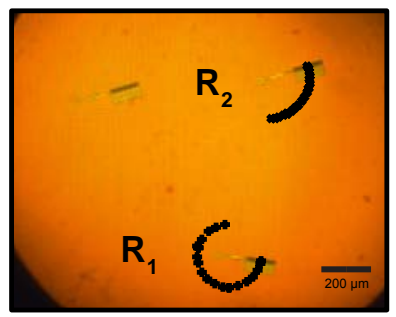

$\mathbf{t}=\mathbf{8} \mathrm{s}$.

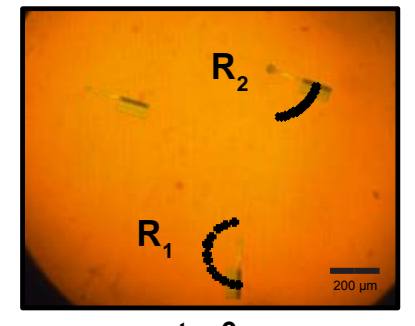

$\mathrm{t}=6 \mathrm{~s}$.

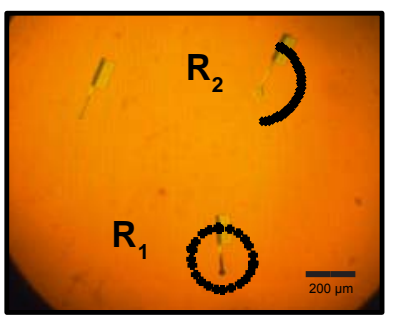

$\mathrm{t}=12 \mathrm{~s}$.

Fig. 13. Optical micrographs of robots $R_{1}$ and $R_{2}$ at times $t=2,6,8,12$ seconds while following turning trajectories. Traces of the completed trajectories for both robots are shown in black.

Fig. 15 shows the implementation of TSC where the center of rotation of one robot is translated in $\mathbb{R}^{2}$ by two independent vectors, analogous to the concept illustrated in Fig. 8 . The center of rotation for robot $R_{2}, c_{r}\left(R_{2}\right)$, is translated by two vectors $\gamma$ and $\gamma_{2}$. The robot $R_{1}$ completes two closed-loop orbits during this experiment. Again, a slight drift in the center of rotation $c_{r}\left(R_{1}\right)$ is apparent due to control error. During the experiment, the robot $R_{2}$ moves partially outside the view of the optical microscope.

Fig 16 shows the implementation of TSC to independently translate the robot with a larger turning rate, analogous to the implementation illustrated in Fig. 9 The steps in this particular implementation were conducted in the reverse order than what was suggested in Sec. III. In this implementation, robot $R_{2}$ was first translated using a combination of turning and straight-line trajectories, displacing the center of rotation

turning trajectory while robot $R_{1}$ completes an orbit. Control error attributed to a slight displacement of the radius of rotation $c_{r}\left(R_{1}\right)$. 


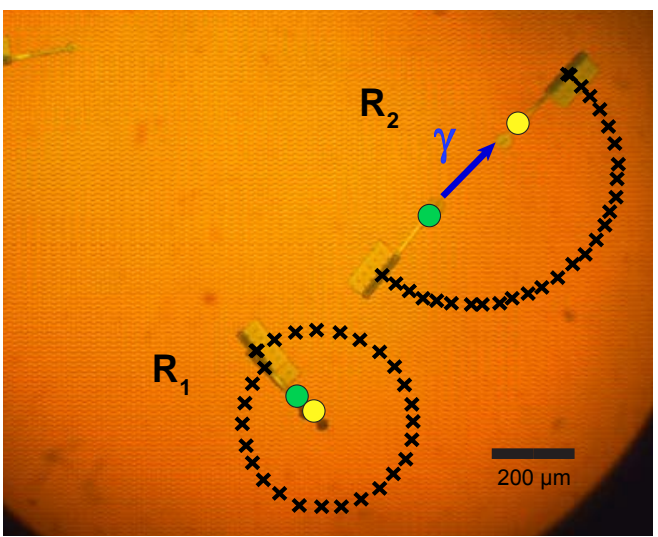

Fig. 14. Composite optical micrograph showing the basic implementation of TSC. Robots $R_{1}$ and $R_{2}$ follow interpolated turning trajectories. As robot $R_{1}$ completes an orbit and returns back to starting configuration, the center of rotation of robot $R_{2}, c_{r}\left(R_{2}\right)$, is translated by a vector $\gamma$.

$c_{r}\left(R_{1}\right)$ by vector $\eta$ (Fig. 16a). Consequently, the center of rotation $c_{r}\left(R_{2}\right)$ was translated back to its starting location, completing a $c_{r}$-orbit for $R_{2}$. Also in this experiment, a slight drift in the location of $c_{r}\left(R_{2}\right)$ can be observed. In practice, this control error will define the ultimate accuracy with which the TSC can be implemented, analogous to forward projections described in [7].

\section{CONCLUSION}

We presented a new method for independent control of stress-engineered MEMS microrobots (MicroStressBots). Called Turning-rate Selective Control (TSC), this method allows for the independent translation of centers of rotation $c_{r}$ of MicroStressBots that are identical except for their turning rates. This allows the individual robots to be maneuvered independently to within the turning radius $(r)$ away from arbitrary locations in $\mathbb{R}^{2}$. The concept of TSC was experimentally validated using physical fabricated MicroStressBots.

The mechanism of TSC is both different from, and independent of, SESat-based control [7]. This allows TSC to augment SESat and increase the number of microrobots that can be independently controlled using a single, global, control signal. When properly designed, $n$ MicroStressBots can be controlled using $2 \sqrt{n}$ independent control voltage levels [7]. Consider a hybrid SESat-TSC $(u)$ system containing $n=u \times w$ robots, where $u$ is the number of groups of $w$ SESat MicroStressBots that are differentiated through their $u$ different turning rates. The $w$ robots within a group are controlled using SESat, while the robots between the $u$ groups are controlled using TSC. Such SESat-TSC $(u)$ system can independently control $n$ robots using the same number of control voltage levels as a $w$-robot SESat system, and has control voltage complexity of $2 \sqrt{\frac{n}{u}}$.

For example, suppose one wants to independently control 100 MicroStressBots through a single global control signal. According to SESat-based control, one would need 20 control voltage levels to achieve independent control. Suppose we divide the 100 microrobots into four groups, each containing

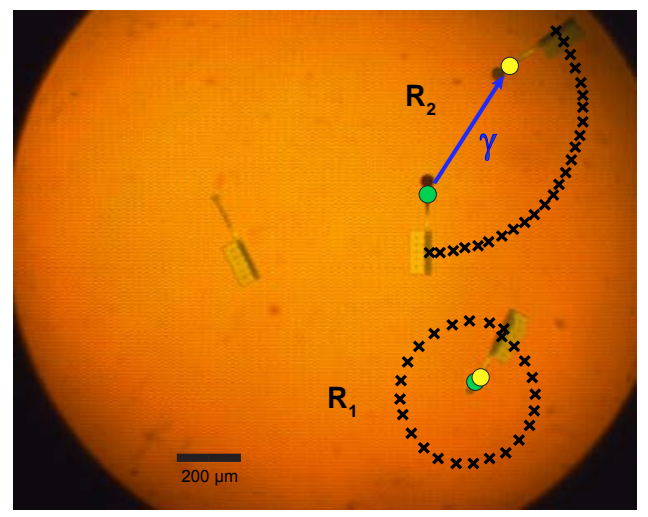

(a)

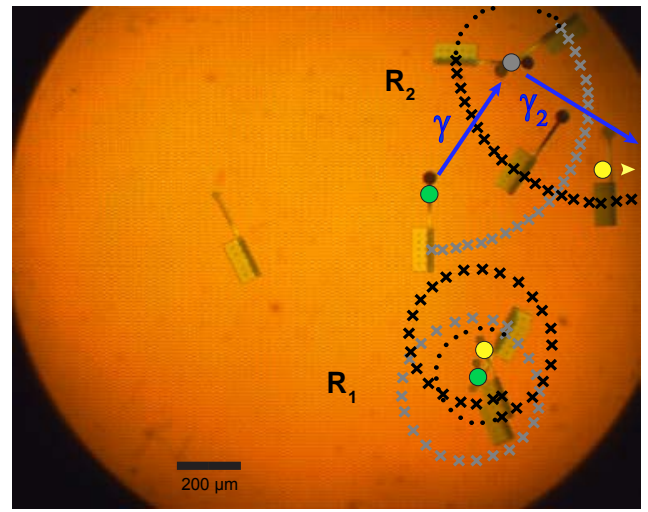

(b)

Fig. 15. Composite optical micrographs showing the translation of the center of rotation for robot $R_{2}, c_{r}\left(R_{2}\right)$, by two linearly independent vectors. (a) Both robots follow interpolated turning trajectories. The center of rotation $c_{r}\left(R_{2}\right)$ is displaced by vector $\gamma$ while robot $R_{1}$ completes an orbit. (b) Both robots turn (turning trajectories are denoted by doted lines). During turning, the centers of rotation of both robots remain in place, allowing the angle between $\gamma$ and $\gamma_{2}$ to be set to an arbitrary value. The center of rotation for robot $R_{2}, c_{r}\left(R_{2}\right)$, is then translated using interpolated turning by a second vector $\gamma_{2}$, as $R_{2}$ moves outside of the view of the microscope (intermediate configurations of robot $R_{2}$ are included for clarity). The yellow dot and arrow point to the approximate location of $c_{r}\left(R_{2}\right)$ after the completion of the experiment.

25 robots that are independently controlled using SESat, while robots between the groups are differentiated using TSC. The robots within this hybrid SESat-TSC(4) system can now be controlled using only 10 independent control voltage levels. Ofcourse, TSC can only maneuver the centers of rotation for the robots in $\mathbb{R}^{2}$. However, the SESat system can decouple the rotation of the individual robots. It would be interesting to analyze whether a SESat-TSC(n) system can be engineered to independently maneuver the robots in $\mathbb{R}^{2} \times \mathbb{S}^{1}$

Because TSC cannot selectively snap-down or release the steering arms of the individual robots, all robots either all move in a straight line or all turn at the same time. Consequently, the orientations $\theta$ of the robots controlled by TSC remain coupled, i.e. cannot be independently controlled. Because $r>0$, the MicroStressBots do not turn in place. To approximate desired configurations in $\mathbb{R}^{2} \times \mathbb{S}^{1}$, the robots must orbit around their centers of rotation $\left(c_{r}\right)$ until mutual 


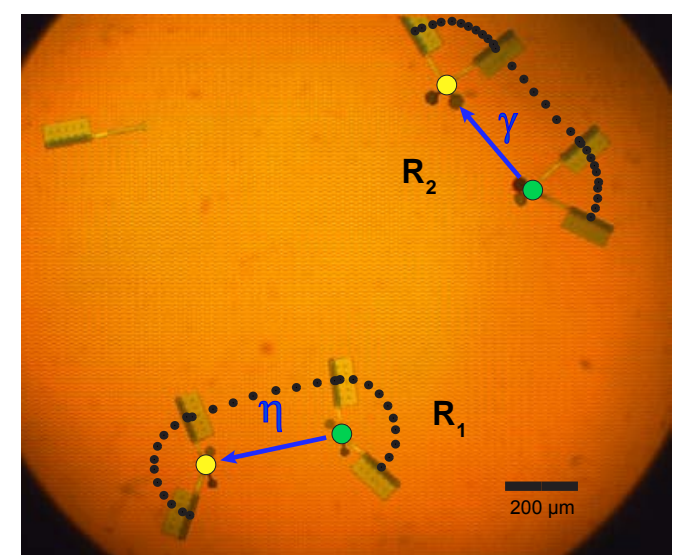

(a)

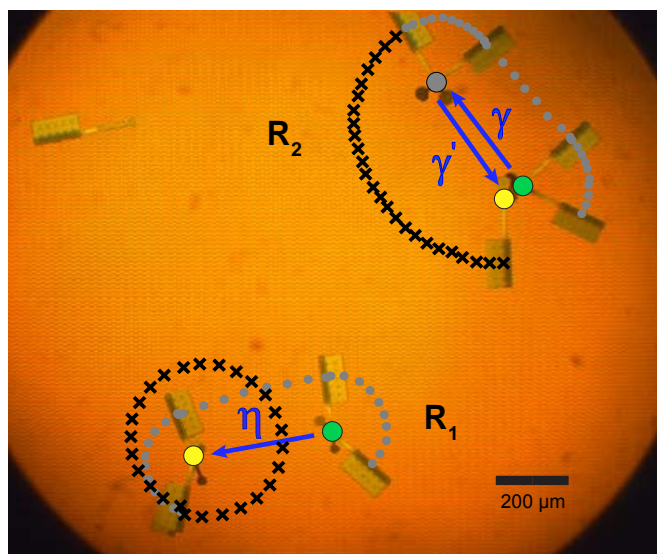

(b)

Fig. 16. Composite optical micrographs showing the implementation of TSC to independently translate the robot with a larger turning rate $\left(R_{1}\right)$. (a) The center of rotation of robot $R_{2}, c_{r}\left(R_{2}\right)$, is translated using a combination of turning and straight-line trajectories (denoted by doted lines), and is translated by a vector $\gamma$. There resulting trajectory of robot $R_{1}$ does not form a an orbit, causing the center of rotation $c_{r}\left(R_{1}\right)$ to be translated by vector $\eta$. (b) The center of rotation of robot $R_{2}, c_{r}\left(R_{2}\right)$, is translated back to its starting location by vector $\gamma^{\prime}$ using interpolated turning, completing a $c_{r}$-orbit for $R_{2}$. Because the interpolated turning trajectory completes a $c_{r}$-orbit for robot $R_{1}$, the center of rotation $c_{r}\left(R_{1}\right)$ maintains its displacement by vector $\eta$ from step (a).

differences in their turning rates cause their orientations to all align close to the desired configurations. Hence, there is a trade-off between the lengths of the robot trajectories and the resulting proximity to the goal configurations, which is especially important when we consider perturbations due to the effects of control error. It would be interesting to fully investigate such trade-offs in future work.

Finally, it is also worth mentioning that interesting related theoretical ideas have been also presented in e.g. [2], and it would be interesting to investigate how these methods fit with the SESat-TSC framework.

\section{ACKNOWLEDGMENTS}

This work was supported by a grant to B.R.D. from the Duke Institute of Brain Sciences, grant numbers GM-65982 to B.R.D. from NIH, and 2000-DT-CX-K001 to B.R.D., from the Office for Domestic Preparedness, Department of Homeland
Security, USA, and funded in part by the Center for Information Technology Research in the Interest of Society (CITRIS). The authors wish to thank D. Balkcom, C. Bailey-Kellogg, and Kris Pister for their advice and suggestions. The electron micrograph was taken at the Dartmouth Ripple Electron Microscopy Laboratory, with the help of C.P. Daghlian.

\section{REFERENCES}

[1] I. V. Borzenets, I. Yoon, M. W. Prior, B. R. Donald, R. D. Mooney, and G. Finkelstein. Ultra-sharp metal and nanotube-based probes for applications in scanning microscopy and neural recording. Journal of Applied Physics, 111(7):074703, 2012.

[2] T. Bretl. Control of many agents using few instructions. In the Proceedings of Robotics: Science and Systems 2007 conference, Atlanta, GA, 2007.

[3] A. Cowen, B. Hardy, R. Mahadevan, and S. Wilcenski. PolyMUMPs Design Handbook. MEMSCAP, 2011.

[4] E. Diller, C. Pawashe, S. Floyd, and M. Sitti. Assembly and disassembly of magnetic mobile micro-robots towards deterministic 2-D reconfigurable micro-systems. The International Journal of Robotics Research, 30(14): 1667-1680, December 2011.

[5] B. R. Donald, C. G. Levey, C. McGray, I. Paprotny, and D. Rus. An untethered, electrostatic, globallycontrollable MEMS micro-robot. Journal of Microelectromechanical Systems, 15(1):1-15, January 2006.

[6] B. R. Donald, C. G. Levey, and I. Paprotny. Planar microassembly by parallel actuation of MEMS microrobots. Journal of Microelectromechanical Systems, 17(4):789808, August 2008.

[7] B. R. Donald, C. G. Levey, I. Paprotny, and D. Rus. Simultaneous control of multiple mems microrobots. In the proceedings of the 9th International Workshop on Algorithmic Foundations of Robotics (WAFR), December 2008, and also in Springer Tracts on Advanced Robotics, 57, G. S. Chirikjian, H. Choset, M. Morales, and T. Murphey (eds.), Springer-Verlag (Berlin), (2010).

[8] S. Floyd, C. Pawashe, and M. Sitti. An untethered magnetically actuated micro-robot capable of motion on arbitrary surfaces. In the Proceedings of IEEE International Conference on Robotics and Automation (ICRA), 2008.

[9] D. R. Frutiger, B. E. Kratochvil, K. Vollmers, and B. J. Nelson. Magmites wireless resonant magnetic microrobots. In the Proceedings of IEEE International Conference on Robotics and Automation (ICRA), May 2008.

[10] A. Ghosh and P. Fischer. Controlled propulsion of artificial magnetic nanostructured propellers. Nano letters, 9 (6):2243-2245, 2009.

[11] S. LaValle. Planning Algorithms. Cambridge University Press, 2006. 